\title{
Article
}

\section{The Hadamard product and recursively defined sequences}

\section{Sergei Dmitrievich Kazenas}

(to Margarita)

\author{
Independent researcher, Moscow, Russia.; kazenas@pm.me
}

Received: 13 December 2019; Accepted: 27 January 2020; Published: 22 February 2020.

\begin{abstract}
In this paper, the approach to obtaining nontrivial formulas for some recursively defined sequences is illustrated. The most interesting result in the paper is the formula for the solution of quadratic map-like recurrence. Also, some formulas for the solutions of linear difference equations with variable coefficients are obtained. At the end of the paper, some integer sequences associated with a quadratic map are considered.
\end{abstract}

Keywords: The Hadamard product, recursively defined sequence, difference equation, quadratic map.

MSC: 39A10, 05A15.

\section{Introduction}

$\mathbf{L}$ et us begin with some notation. Let $\mathrm{b}$ be a row vector and a be a finite row vector; $(\mathrm{b})_{j}$ denotes $j$ th element of vector $\mathrm{b},|\mathrm{b}|$ denotes length of $\mathrm{b} ; \mathrm{a} \cup \mathrm{b} \stackrel{\text { def }}{=}\left((\mathrm{a})_{1}, \ldots,(\mathrm{a})_{|\mathrm{a}|},(\mathrm{b})_{1}, \ldots\right),(\mathrm{b})_{l}^{m} \stackrel{\text { def }}{=}\left((\mathrm{b})_{j}\right)_{j=l^{\prime}}^{m}$ $|\mathrm{b}|{ }_{x} \stackrel{\text { def }}{=} \sum_{j=1}^{|\mathrm{b}|}(\mathrm{b})_{j} x^{j-1}, 1_{m} \stackrel{\text { def }}{=}(1)_{j=1}^{m}, 0_{m} \stackrel{\text { def }}{=}(0)_{j=1}^{m},[l, m]_{j} \stackrel{\text { def }}{=}\left(1_{\infty} \times\left(0_{l} \cup 1_{m}\right)\right)_{j}$, where $\times$ denotes the Kronecker product, $l \in \mathbb{N}, m \in \mathbb{N} \cup\{\infty\}$. Note that the last function can be expressed by the ceiling function: $[l, m]_{j}=$ $\lceil(j+m) /(l+m)\rceil-\lceil j /(l+m)\rceil$.

Also, we use ordinary notation to denote the corresponding entrywise operations. For example, $\mathrm{a}^{2}$ expresses the Hadamard square: $\mathrm{a}^{2}=\left((\mathrm{a})_{j}^{2}\right)_{j=1}^{|\mathrm{a}|}$.

It should be noted that there are many papers on sequences generated by linear difference equations with variable coefficients. See, for examle, [1-3]. The simple approach illustrated here involves constructing for each such sequence a corresponding recursive vector sequence, which can be explicitly expressed using the following property of Hadamard product: $(\mathrm{ac}) *(\mathrm{bd})=(\mathrm{a} * \mathrm{~b})(\mathrm{c} * \mathrm{~d})$, where $|\mathrm{c}|=|\mathrm{a}|,|\mathrm{d}|=|\mathrm{b}|$ and $* \in\{\smile, \times\}$.

\section{Linear recurrences}

First we use this property with respect to concatenation.

Theorem 1. If $x_{1}, x_{2}$ are arbitrary numbers, $a_{n}, b_{n}$ are arbitrary number sequences and $x_{n}=a_{n} x_{n-1}+b_{n} x_{n-2}$ for $n \geqslant 3$, then

$$
x_{n}=\sum_{j=1}^{f_{n}}\left(\left(x_{1}-x_{2}\right)(\mathrm{f})_{j}+x_{2}\right)\left(\prod_{\substack{3 \leqslant k \leqslant n \\\left(\mathrm{f}_{k}\right)_{j}=1}} b_{k}\right)\left(\prod_{\substack{3 \leqslant k \leqslant n \\\left(\mathrm{f}_{k}+\mathrm{f}_{k+1}\right)_{j}=0}} a_{k}\right),
$$

where $f_{n}$ is $n$th Fibonacci number, $\mathrm{f}=(0,1,0,0, \ldots)$ is infinite Fibonacci word; $\mathrm{f}_{k}$ is obtained from $\mathrm{f}$ by replacing each entry of zero with $f_{k-1}$ zeros and each entry of one with $f_{k-2}$ ones.

Proof. Define vectors: $\mathrm{x}_{1}=\left(x_{1}\right), \mathrm{x}_{2}=\left(x_{2}\right), \mathrm{x}_{n}=\left(b_{n} \mathrm{x}_{n-2}\right) \smile\left(a_{n} \mathrm{x}_{n-1}\right)$ for $n \geqslant 3$ and $\mathrm{y}_{1}=\mathrm{x}_{1}, \mathrm{y}_{n}=\mathrm{y}_{n-1} \triangleleft \mathrm{x}_{n}$, $\mathrm{p}_{n}=1_{f_{n+1}-1} \cup\left(b_{n} 1_{f_{n-2}}\right) \cup\left(a_{n} 1_{f_{n-1}}\right)$ for $n \geqslant 2$.

Let $\Lambda_{k} \mathrm{~b} \stackrel{\text { def }}{=} \mathrm{b}_{\smile}(\mathrm{b})_{k}^{|\mathrm{b}|}$. We have $\mathrm{y}_{n}=\mathrm{p}_{n} \Lambda_{f_{n-1}} \mathrm{y}_{n-1}$ for $n \geqslant 3$, from which it follows that $\mathrm{y}_{n}=\mathrm{y}_{2, n} \prod_{k=3}^{n} \mathrm{p}_{k, n}$ where $\mathrm{y} 2, n=\Lambda_{f_{n-1}} \Lambda_{f_{n-2}} \ldots \Lambda_{f_{2}} \mathrm{y}_{2}, \mathrm{p}_{k, n}=\Lambda_{f_{n-1}} \Lambda_{f_{n-2}} \ldots \Lambda_{f_{k}} \mathrm{p}_{k}$. 
Partition $\mathrm{y}_{2, n}=\mathrm{y}_{1}^{\prime} \smile \ldots \smile \mathrm{y}_{n}^{\prime}$ such that $\left|\mathrm{y}_{i}^{\prime}\right|=f_{i}$, then $\mathrm{y}_{1}^{\prime}=\mathrm{x}_{1}, \mathrm{y}_{2}^{\prime}=\mathrm{x}_{2}, \mathrm{y}_{n}^{\prime}=\mathrm{y}_{n-2}^{\prime}+\mathrm{y}_{n-1}^{\prime}$ for $n \geqslant$ 3. Similarly partition $\mathrm{p}_{k, n}=\mathrm{p}_{k, 1}^{\prime} \smile \cdots \cup \mathrm{p}_{k, n}^{\prime}$ such that $\left|\mathrm{p}_{k, i}^{\prime}\right|=f_{i}$, then $\mathrm{p}_{k, i}^{\prime}=1_{f_{i}}$ for $1 \leqslant i \leqslant k-1, \mathrm{p}_{k, k}^{\prime}=$ $\left(b_{k} 1_{f_{k-2}}\right) \cup\left(a_{k} 1_{f_{k-1}}\right), \mathrm{p}_{k, i}^{\prime}=\mathrm{p}_{k, i-2}^{\prime} \sim \mathrm{p}_{k, i-1}^{\prime}$ for $k+1 \leqslant i \leqslant n$.

Note that $\left(\mathrm{x}_{n}\right)_{-}=\left(\mathrm{y}_{n}^{\prime}\right)_{-} \prod_{k=3}^{n}\left(\mathrm{p}_{k, n}^{\prime}\right)_{-}$, where by $(\mathrm{a})_{-}$we denote the vector composed of elements of a in reverse order. Now $\left(\mathrm{y}_{n}^{\prime}\right)_{-}$and $\left(\mathrm{p}_{k, n}^{\prime}\right)$ - can be expressed in terms of infinite generalized Fibonacci words: $\left(\mathrm{y}_{n}^{\prime}\right)_{-}=\left(x_{1}-x_{2}\right)(\mathbf{f})_{1}^{f_{n}}+x_{2} 1_{f_{n}},\left(\mathrm{p}_{k, n}^{\prime}\right)_{-}=\left(\mathrm{f}_{k+1}+b_{k} \mathrm{f}_{k}+a_{k}\left(1_{f_{n}}-\mathrm{f}_{k+1}-\mathrm{f}_{k}\right)\right)_{1}^{f_{n}}$.

Finally using $x_{n}=\left|\left(\mathrm{x}_{n}\right)_{-}\right|_{1}$ we get the result.

The same sequence can be expressed with help of the Kronecker product.

Theorem 2. If $x_{1}, x_{2}$ are arbitrary numbers, $a_{n}, b_{n}$ are arbitrary number sequences and $x_{n}=a_{n} x_{n-1}+b_{n} x_{n-2}$ for $n \geqslant 3$, then

$$
x_{n}=\sum_{\substack{2^{n-2}+1 \leqslant j \leqslant 2^{n-1} \\ \vartheta\left(2^{n-1}-j+1\right)=1}}\left(\left(x_{2}-x_{1}\right)[1,1]_{j}+x_{1}\right) \prod_{\substack{0 \leqslant k \leqslant n-3 \\\left[3 \cdot 2^{k}, 2^{k}\right]_{j}=1}} a_{k+3} \prod_{\substack{0 \leqslant k \leqslant n-3 \\\left[2^{k}, 2^{k}\right]_{j}=0}} b_{k+3},
$$

where $\vartheta(n) \stackrel{\text { def }}{=} \prod_{k=0}^{\infty}\left(1-\left[3 \cdot 2^{k}, 2^{k}\right]_{n}\right)$.

Proof. Define vectors: $\mathrm{r}_{1}=\left(x_{1}, x_{2}\right), \mathrm{r}_{n}=\left(1_{2} \times \mathrm{r}_{n-1}\right)\left(\mathrm{h}_{n} \times 1_{2^{n-2}}\right)$ for $n \geqslant 2$, where $\mathrm{h}_{n}=\left(0,1, b_{n+1}, a_{n+1}\right)$. It can be easily shown that $\left|\left(r_{n}\right)_{2^{n-1}+1}^{2^{n}}\right|_{1}=x_{n+1}$. Solving the recurrence equation we get: $r_{n}=\left(1_{2^{n-1}} \times\right.$ $\left.\mathrm{r}_{1}\right) \prod_{k=2}^{n}\left(1_{2^{n-k}} \times \mathrm{h}_{k} \times 1_{2^{k-2}}\right)$. Taking into account that $\mathrm{h}_{k}=(0,1,1,1)\left(b_{k+1}, 1, b_{k+1}, 1\right)\left(1,1,1, a_{k+1}\right)$ and doing some calculations we get the result.

The following lemma allows us to generalize the result to the nonhomogeneous case.

Lemma 1. If $\mathrm{x}_{1}$ is arbitrary vector, $\mathrm{b}_{n}$ is 0,1 -vector sequence, $\mathrm{a}_{n}$ and $\mathrm{c}_{n}$ are such that $\left|\mathrm{a}_{n}\right|=\left|\mathrm{c}_{n}\right|=\left|\mathrm{x}_{1}\right| \prod_{i=2}^{n}\left|\mathrm{~b}_{i}\right|$; $\mathrm{x}_{n}=\mathrm{a}_{n}\left(\mathrm{~b}_{n} \times \mathrm{x}_{n-1}\right)+\mathrm{c}_{n}$ for $n \geqslant 2$, then

$$
\mathrm{x}_{n}=\left(\mathrm{b}_{n, 2} \times \mathrm{x}_{1}\right) \prod_{k=2}^{n}\left(\mathrm{~b}_{n, k+1} \times \mathrm{a}_{k}\right)+\sum_{i=2}^{n}\left(\mathrm{~b}_{n, i+1} \times \mathrm{c}_{i}\right) \prod_{k=i+1}^{n}\left(\mathrm{~b}_{n, k+1} \times \mathrm{a}_{k}\right),
$$

where $\mathrm{b}_{n, k}=\mathrm{b}_{n} \times \mathrm{b}_{n-1} \times \cdots \times \mathrm{b}_{k}$, if $k \leqslant n$ and $\mathrm{b}_{n, k}=1_{1}$, if $k>n$.

The proof is straightforward.

Vectors $r_{n}^{\prime}$ for similar nonhomogeneous sequence $x_{n}^{\prime}=a_{n} x_{n-1}^{\prime}+b_{n} x_{n-2}^{\prime}+c_{n}$ such that $\left|\left(r_{n}^{\prime}\right)_{2^{n-1}+1}^{2^{n}}\right|_{1}=$ $x_{n+1}^{\prime}$, are defined as follows: $\mathrm{r}_{1}^{\prime}=\left(x_{1}, x_{2}\right), \mathrm{r}_{n}^{\prime}=\left(1_{2} \times \mathrm{r}_{n-1}^{\prime}\right)\left(\mathrm{h}_{n} \times 1_{2^{n-2}}\right)+c_{n}\left(0_{2^{n}-1} 1_{1}\right)$ for $n \geqslant 2$. To use the lemma we should, of course, do substitutions $\mathrm{a}_{n}=\mathrm{h}_{n} \times 1_{2^{n-2}}$ and $\mathrm{b}_{n, k}=1_{2^{n-k+1}}$.

Theorem 3. If $w_{0}$ is arbitrary number, $a_{n, j}$ is arbitrary number sequence and $w_{n}=\sum_{j=0}^{n-1} a_{n, j} w_{j}$ for $n \in \mathbb{N}$, then

$$
\sum_{j=0}^{n} w_{j}=w_{0} \sum_{\mathrm{v} \in \mathbb{V}_{n}} \prod_{k=1}^{|\mathrm{v}|} a_{(\mathrm{v})_{k},\left(0_{1} \triangleleft \mathrm{v}\right)_{k^{\prime}}}
$$

where set $\mathbb{V}_{n}$ consists of all vectors $\mathrm{v}$ such that $1 \leqslant(\mathrm{v})_{i-1}<(\mathrm{v})_{i} \leqslant n$ for $2 \leqslant i \leqslant|\mathrm{v}|$.

Proof. Define vectors: $\mathrm{w}_{0}=\left(w_{0}\right), \mathrm{w}_{1}=\left(w_{0}, a_{1,0} w_{0}\right), \mathrm{w}_{n}=\mathrm{w}_{n-1} \cup\left(\mathrm{w}_{n-1} \mathrm{q}_{n}\right)$ for $n \geqslant 2$, where $\left(\mathrm{q}_{n}\right)_{1}=a_{n, 0}$, $\left(\mathrm{q}_{n}\right)_{2^{k-1}+1}^{2^{k}}=a_{n, k} 1_{2^{k-1}}$ for $1 \leqslant k \leqslant n-1$. From the recurrence equation it follows that if equality $\left|\mathrm{w}_{l}\right|_{1}=\sum_{j=0}^{l} w_{j}$ is true for $l=n-1 \geqslant 1$, then it is true for $l=n$; it is true for $l=1$, so we conclude that it is true for any $l \in \mathbb{N}$. Solving the recurrence equation, we get: $\mathrm{w}_{n}=w_{0} \prod_{k=1}^{n}\left(1_{2^{n-k}} \times\left(1_{2^{k-1}} \triangleleft \mathrm{q}_{k}\right)\right)$ for $n \in \mathbb{N}$. Noting that $\mathrm{q}_{k}=\left(a_{k,\left\lceil\log _{2} j\right\rceil}\right)_{j=1}^{2^{k-1}}$, we have

$$
\left|\mathrm{w}_{n}\right|_{1}=w_{0} \sum_{j=1}^{2^{n}} \prod_{k=1}^{n}\left(\left[2^{k-1}, 2^{k-1}\right]_{j}\left(a_{k,\left[\log _{2}\left(1+(j-1) \bmod 2^{k-1}\right)\right\rceil}-1\right)+1\right) .
$$


The quantity $\left[2^{k}, 2^{k}\right]_{j}$ equals the value of $k$ th digit of number $(j-1)$ in binary numeral system. If $k_{i}$ is serial number of $i$ th digit 1 , then $\left\lceil\log _{2}\left(1+(j-1) \bmod 2^{k_{i}}\right)\right\rceil=1+k_{i-1}$. Therefore, $\left|\mathrm{w}_{n}\right|_{1}=w_{0} \sum_{j} \prod_{i} a_{k_{i}+1, k_{i-1}+1}$, assuming $k_{0}=-1$. Here $\left(k_{i}+1\right)$ ranges over $\mathrm{v} \in \mathbb{V}_{n}: k_{i}+1=(\mathrm{v})_{i}$.

In the same way vectors $\mathrm{w}_{n}^{\prime}$ for nonhomogeneous sequence $w_{n}^{\prime}=c_{n}+\sum_{j=0}^{n-1} a_{n, j} w_{j}^{\prime}$ such that $\left|\mathrm{w}_{l}^{\prime}\right|_{1}=$ $\sum_{j=0}^{l} w_{j}$, are defined as follows: $\mathrm{w}_{0}^{\prime}=\left(w_{0}\right), \mathrm{w}_{1}^{\prime}=\left(w_{0}, c_{1}+a_{1,0} w_{0}\right), \mathbf{w}_{n}^{\prime}=\mathrm{w}_{n-1}^{\prime} \smile\left(\mathrm{w}_{n-1}^{\prime} \mathrm{q}_{n}\right)+c_{n}\left(0_{2^{n}-1} 1_{1}\right)$ for $n \geqslant 2$.

\section{Quadratic map}

It is well-known that in many cases iterations of a polynomial of degree 2 in the general case, i.e. solutions of quadratic map, can be expressed by iterations of a polynomial of degree 2 with one parameter.

Theorem 4. Let $p^{(0)}(x)=x, p^{(n)}(x)=p^{(n-1)}(p(x))($ for $n \in \mathbb{N})$ be iterations of polynomial $p(x)=\lambda(x+1) x$, then

$$
p^{(n)}(x)=\sum_{k=1}^{2^{n}} x^{k} \sum_{i=(k-1) \omega_{n}}^{k \omega_{n}-1} \prod_{j=1}^{n} \lambda^{\mu_{i, j-1}}\left(\begin{array}{c}
\mu_{i, j-1} \\
\mu_{i, j}-\mu_{i, j-1}
\end{array}\right),
$$

where $\omega_{n}=2^{\frac{n(n-1)}{2}}, \mu_{i, j}=\left\lceil\frac{1+i \bmod \omega_{j+1}}{\omega_{j}}\right\rceil$.

Proof. Any polynomial $p^{(n)}(x)$ can be expressed as follows: $p^{(n)}(x)=\sum_{k=1}^{2^{n}} g_{n, k} x^{k}$, where $g_{n, k}=g_{n, k}(\lambda)$ are polynomials defined by equalities: $g_{0,1}=1, g_{n, k}=\sum_{i=1}^{2^{n-1}} q_{k, i} g_{n-1, i}\left(\right.$ for $1 \leqslant k \leqslant 2^{n}$ ), where $q_{k, i}=\lambda^{i}\left(\begin{array}{c}i \\ k-i\end{array}\right)$.

Let $\mathrm{p}_{0}=(1), \mathrm{p}_{n}=\left(1_{2^{n}} \times \mathrm{p}_{n-1}\right)\left(\mathrm{q}_{n} \times 1_{\omega_{n-1}}\right)$ for $\in \mathbb{N}$, where

$$
\mathrm{q}_{n}=\left(q_{1, j}\right)_{j=1}^{2^{n-1}} \smile\left(q_{2, j}\right)_{j=1}^{2^{n-1}} \smile \cdots \smile\left(q_{2^{n}, j}\right)_{j=1}^{n^{n-1}}=\left(q_{\left\lceil i / 2^{n-1}\right\rceil, 1+(i-1) \bmod 2^{n-1}}\right)_{i=1}^{2^{2 n-1}} .
$$

Then $\left|\left(\mathrm{p}_{n}\right)_{1+(k-1) \omega_{n}}^{k \omega_{n}}\right|_{1}=g_{n, k}$. Solving the equation, we get:

$$
\mathrm{p}_{n}=\prod_{k=1}^{n}\left(1_{2}^{\frac{(n+k+1)(n-k)}{2}} \times \mathrm{q}_{k} \times 1_{\omega_{k-1}}\right) .
$$

The last step is to check that $1_{\infty} \times \mathrm{q}_{k} \times 1_{\omega_{k-1}}=\left(\mathrm{q}_{\mu_{j, k}, \mu_{j, k-1}}\right)_{j=0}^{\infty}$.

Remark 1. Using generating polynomial $\left|\mathrm{q}_{k}\right|_{t}=\lambda\left(1+t^{m}\right)\left(\left(\lambda t^{m+1}+\lambda t^{2 m+1}\right)^{m}-1\right) /\left(\lambda t^{m+1}+\lambda t^{2 m+1}-1\right)$, where $m=2^{k-1}$ and taking in account formula $|\mathrm{a} \times \mathrm{b}|_{t}=|\mathrm{a}|_{t \mid \mathrm{b}}|\mathrm{b}|_{t}$ we can represent $p^{(n)}(x)$ as Hadamard's product of $n$ functions. The polynomial $\left|q_{k}\right|_{t}$ can be derived using polynomials $\varphi_{k, m}=\sum_{j=1}^{m}\left(\begin{array}{c}j \\ k-j\end{array}\right)(\lambda t)^{j-1}$ as follows. Taking in account that $\varphi_{k, m}=\lambda t\left(\varphi_{k-1, m}+\varphi_{k-2, m}\right)-(\lambda t)^{m}\left(\begin{array}{c}m+1 \\ k-m-1\end{array}\right)$ for $k \geqslant 3$ and $m \geqslant 1$, we can write

$$
\begin{aligned}
\lambda^{-1}\left|\mathrm{q}_{k}\right|_{t} & =\sum_{k=1}^{2 m} t^{(k-1) m} \varphi_{k, m}=1+t^{m}+\lambda t^{m+1} \sum_{k=1}^{2 m-1} t^{(k-1) m} \varphi_{k, m}+\lambda t^{2 m+1} \sum_{k=1}^{2 m-2} t^{(k-1) m} \varphi_{k, m}-\lambda^{m} \sum_{k=2}^{2 m} t^{k m}\left(\begin{array}{c}
m+1 \\
k-m-1
\end{array}\right) \\
& =1+t^{m}+\lambda t^{m+1}\left(\lambda^{-1}\left|\mathrm{q}_{k}\right|_{t}-t^{m(2 m-1)} \varphi_{2 m, m}\right)+\lambda t^{2 m+1}\left(\lambda^{-1}\left|\mathrm{q}_{k}\right|_{t}-t^{m(2 m-1)} \varphi_{2 m, m}-t^{m(2 m-2)} \varphi_{2 m-1, m}\right) \\
& -\lambda^{m}\left(t^{m(m+1)}\left(1+t^{m}\right)^{m+1}-\left(m+t^{m}+1\right) t^{2 m^{2}+m}\right) .
\end{aligned}
$$

From here taking in account $\varphi_{2 m, m}=(\lambda t)^{m-1}$ and $\varphi_{2 m-1, m}=m(\lambda t)^{m-1}$ we immediately get $\left|q_{k}\right|_{t}$.

Let's consider another episode. Let $s^{(0)}(x)=x, s^{(n)}(x)=s^{(n-1)}(s(x))$ (for $n \in \mathbb{N}$ ) be iterations of polynomial $s(x)=s_{\lambda}(x)=\lambda\left(x^{2}-1\right)+1$ and $\lambda \neq 0$. Define vectors: $\mathbf{s}_{1}=(x-1,1), \mathrm{s}_{n}=$ $\lambda \mathbf{s}_{n-1}^{\langle 2\rangle}-(\lambda-1)\left(0_{2^{2^{n-1}-1}} \smile 1\right)$ for $n \geqslant 2$, where triangular brackets indicate Kronecker degree. Obviously, $\left|\mathbf{s}_{n}\right|_{1}=s\left(\left|\mathbf{s}_{n-1}\right|_{1}\right)=s^{(n)}(x)$. 
Solving the equation, we get:

$$
\mathbf{s}_{n}=\lambda \mathbf{s}_{n-1}^{\langle 2\rangle} \mathbf{I}_{n-1}=\lambda^{2^{n-1}-1} \mathbf{s}_{1}^{\left\langle 2^{n-1}\right\rangle} \mathbf{r}_{\lambda, n-1}=\lambda^{2^{n-1}-1} \mathbf{s}_{1}^{\left\langle 2^{n-1}\right\rangle}(\mathbf{r}-)_{1}^{2^{2^{n-1}}}
$$

where $\mathrm{I}_{n}=1_{2^{2^{n}}-1} \smile\left(\lambda^{-1}\right), \mathbf{r}_{\lambda, n}=\prod_{i=1}^{n} \mathbf{I}_{i}^{\left\langle 2^{n-i}\right\rangle}, \mathbf{r}-=\prod_{i=1}^{\infty} \mathrm{I}_{i}^{\langle\infty\rangle}$.

Therefore, we have

$$
s_{\lambda}^{(n)}(x)=\lambda^{2^{n-1}-1} \sum_{j=1}^{2^{2^{n}}}(x-1)^{\left(\mathrm{h}_{n}\right)_{j}} \lambda^{-\left(\log _{2} \mathrm{r}_{2, n-1}\right)_{j}}
$$

where $\mathrm{h}_{n}=\log _{2}(2,1)^{\left\langle 2^{n-1}\right\rangle}$. And it can be easily shown that

$$
\left(\mathrm{h}_{n}\right)_{j}=2^{n-1}-\sum_{k=0}^{\infty}\left[2^{k}, 2^{k}\right]_{j} \text { and }\left(\log _{2} \mathrm{r}_{2, n}\right)_{j}=\sum_{k, i=0}^{\infty}\left[2^{2^{i} k}\left(2^{2^{i}}-1\right), 2^{2^{i} k}\right]_{j}
$$

by using simple formula

$$
\left(\log _{2}\left(1_{m-1} \smile(2)\right)^{\langle n\rangle}\right)_{j}=\sum_{k=0}^{n-1}\left[m^{k}(m-1), m^{k}\right]_{j}\left(\text { for } j \leqslant m^{n}\right) .
$$

Substituting 2 for $x$ in (2), we have

$$
s_{\lambda}^{(n)}(2)=\lambda^{2^{n-1}-1} \sum_{k=1}^{2^{n-1}} \kappa_{n, k} \lambda^{-k}
$$

where $\kappa_{n, k}$ denotes the number of elements in $\log _{2} r_{2, n-1}$ that equal to $k$. This function can be defined recursively as follows:

$$
\kappa_{n, 0}=3^{2^{n-1}}, \kappa_{1, k}=\delta_{k, 1}, \kappa_{n, k}=\delta_{k, 2^{n-1}}-\delta_{k, 2^{n-2}}+\sum_{i=0}^{k} \kappa_{n-1, k-i} \kappa_{n-1, i} .
$$

Evaluating $\kappa_{n, k}$ we have:

$$
\kappa_{n, 1}=2^{n-1} 3^{2^{n-1}-1}, \kappa_{n, 2}=-\kappa_{n, 1}\left(\frac{1}{12}-\sum_{i=0}^{n-1} \kappa_{i+1,1}\left(\kappa_{i, 1}^{2}+\delta_{k, 2^{n-1}}-\delta_{k, 2^{n-2}}\right)\right.
$$

and so on.

Remark 2. Replacing $2^{n-1}$ by $n$ in the last expression of (1), we get new sequence of vectors: $\mathbf{s}_{n}^{\prime}=$ $\lambda^{n-1} \mathbf{s}_{1}^{\langle n\rangle}(\mathrm{r})_{1}^{2^{n}}$. Let $f_{n}(x)=\left|\mathbf{s}_{n}^{\prime}\right|_{1}$. Conjecture:

$$
f_{n}(x)=\left\{\begin{array}{l}
f_{n / 2}(s(x)), \text { if } n \text { is even } \\
\lambda x f_{n-1}(x), \text { if } n \text { is odd } \\
x, \text { if } n=1
\end{array}\right.
$$

Conflicts of Interest: "The author declares no conflict of interest."

\section{References}

[1] Popenda, J. (1987). One expression for the solutions of second order difference equations. Proceedings of the American Mathematical Society, 100(1), 87-93.

[2] Kittappa, R. K. (1993). A representation of the solution of the nth order linear difference equation with variable coefficients. Linear algebra and its applications, 193, 211-222. 
[3] Mallik, R. K. (2000). On the solution of a linear homogeneous difference equation with variable coefficients. SIAM Journal on Mathematical Analysis, 31(2), 375-385.

[4] Allouche, J. P., \& Shallit, J. (2003). Automatic sequences: theory, applications, generalizations. Cambridge university press.

[5] Lando, S. K. (2003). Lectures on generating functions (Vol. 23). American Mathematical Society.

[6] Voevodin, V., \& Kuznetsov Y. (1984). Matrices and calculations. Nauka (in Russian).

[7] Fomin, S. (1975). Number systems. The University of Chicago Press.

(c) 2020 by the author; licensee PSRP, Lahore, Pakistan. This article is an open access article distributed under the terms and conditions of the Creative Commons Attribution (CC-BY) license (http://creativecommons.org/licenses/by/4.0/). 